\title{
Clinicopathological nomograms model to predict long-term overall survival and cancer-specific survival in skin cutaneous melanoma: a population-based study
}

\author{
Lingkai Su${ }^{1}$, Jukun Song ${ }^{2}$, Zhuhui Duan ${ }^{1}$, Chen Zhu ${ }^{3}$, Juxiang Peng ${ }^{3}$, Xiongfei Sun ${ }^{4}$, \\ Wei Yư ${ }^{5}$, Xinhai Yin ${ }^{6}$, Jianguo Zhu' ${ }^{6}$ and Hui Chen ${ }^{1}$ \\ ${ }^{1}$ Affiliated Hospital of Stomatology, Medical College, Zhejiang University, Hangzhou, P.R. China \\ ${ }^{2}$ Department of Oral and Maxillofacial Surgery, Guizhou Provincial People's Hospital, Guizhou, China \\ ${ }^{3}$ Guiyang Hospital of Stomatology, Medical College, Guiyang, P.R.China \\ ${ }^{4}$ The Second Clinical Medical College of Jinan University, Guizhou, China \\ ${ }^{5}$ Renmin Hospital, Hubei University of Medicine, Hubei, China \\ ${ }^{6}$ Department of Urology, Guizhou Provincial People's Hospital, Guizhou, China
}

Correspondence to: Hui Chen, email: huiczju66@zju.edu.cn

Keywords: skin cutaneous melanoma; nomograms

Received: July 04, 2017 Accepted: October 30, $2017 \quad$ Published: January 02, 2018

Copyright: Su et al. This is an open-access article distributed under the terms of the Creative Commons Attribution License 3.0 (CC BY 3.0), which permits unrestricted use, distribution, and reproduction in any medium, provided the original author and source are credited.

\section{ABSTRACT}

Skin cutaneous melanoma (SCM) is one of the most aggressive skin cancers with a high mortality rate and the fastest growing global incidence rate of all malignancies. The study was aimed to develop clinical nomograms to predict longterm overall survival and cancer-specific survival (CSS) in patients with SCM. Data of patients diagnosed between 2003 and 2013 were retrieved from the Surveillance, Epidemiology, and End Results. The patients were randomly divided into the Training $(70 \%, n=22,101)$ and Validation $(30 \%, n=9,479)$ cohorts. The probability of CSS and death from other causes was calculated by the competing risk regression model.

Out of 31,580 patients, 4865 died from skin cutaneous melanoma and 2215 died from other causes. The 3- and 5-year probabilities of overall death were 0.671 and 0.865 in the training cohorts, respectively. The 3 - and 5-year probabilities of specific death were 0.410 and 0.506 in the training cohorts, respectively. Univariate and multivariate analyses were employed to choose the independent prognosis variable for OS and CSS. Nomogram models predicting the overall survival and CSS were established according to 14 clinicopathologic characteristics (age at the diagnosis, race, sex, tumor location, tumor histology, TNM stage, Breslow thickness, Clark level, tumor ulceration, tumor size, radiotherapy, and surgery of primary site), with high concordance indexes in both internal validation ( 0.860 for OS and 0.901 for CSS) and external validation ( 0.859 for OS and 0.904 for CSS).

The nomogram model could accurately estimate probabilities of OS and CSS for patients with SCM. The established nomograms could help clinicians in screening patients with high risk of SCM and facilitate individualized treatment.

\section{INTRODUCTION}

Skin cutaneous melanoma (SCM) is an aggressive form of skin cancer with a high mortality rate and the fastest growing global incidence rate of all malignancies
[1, 2]. In 2017 , a total of 87,110 estimated new cases of SCM were diagnosed with 9,730 estimated deaths in the USA alone, making SCM the sixth leading cause of cancer deaths in men and the seventh in women. An estimated 74,640 cases of melanoma was in situ predicted for 2017 
[3]. Considering clinicopathologic heterogeneity and biological behavior diversity, the SCM incidence rates range from $25 \%$ to $61 \%$ among men and $11 \%$ to $43 \%$ among women in the United States, and the mortality rates range from $10 \%$ to $26 \%$; the 5 -year survival rates of SCM in the United States were $78 \%$ for men and $86 \%$ for women $[4,5]$. Therefore, accurate estimates of the prognosis of patients with SCM on the basis of clinicopathologic characteristics would help physicians provide appropriate individualized treatment. Patients with SCM are also at high risk of death from other factors such as distant metastasis, secondary cancers, and chemoradiotherapeutic toxicity. As a result, overall survival might fail to accurately describe a patient's survival rate attributed to SCM, whereas estimated specific death may more accurately describe patient survival due to SCM. Therefore, competing causes of death should not be considered when evaluating cancer-specific survival (CSS) of SCM.

This study aimed to use a large, retrospective population-based database to evaluate and estimate the probability of cancer-specific death and competing risk analysis. This study also developed SCM nomograms to predict long-term OS and CSS probabilities on the basis of multiple clinicopathologic risk factors to improve estimated individual patient treatments and prognosis.

\section{MATERIALS AND METHODS}

\section{Ethics statement}

The study was approved by the Ethical Committee of GuiZhou People's Hospital. Informed patient consent was not required for the data released by the Surveillance, Epidemiology, and End Results (SEER) database.

\section{Data source and patient selection criteria}

Given that TNM stage information was not obtained until 2003, we used the SEER database of the National Cancer Institute (http://seer.cancer.gov/) to screen all patients with SCM diagnosed between 2003 and 2013, which included cancer data from 17 population-based registries among 14 states across the United States, comprising approximately $28 \%$ of the United States population $[6,7]$. The specific inclusion criteria used to identify eligible patients were as follows: 1 ) patients were diagnosed from 2003 to 2013; 2) CS Schema v0204+ was skin cutaneous melanoma; 3 ) histological type ICD-O-3 was limited to $8720 / 3$ (malignant melanoma, NOS); 8721/3 (nodular melanoma); 8742/3 (lentigo malignant, melanoma); 8743/3 (superficial spreading melanoma); 8744/3 (acral lentiginous melanoma, malignant); 8745/3 (desmoplastic melanoma, malignant); and 8772/3 (spindle cell melanoma, NOS); and 4) the SCM primary tumor site was the head and neck (skin of lip, scalp and neck, external ear, other/unspecific parts of face, and eyelid), trunk (skin of trunk), limbs (skin of lower limb and hip, upper limb, and shoulder), and genitals (skin of labium minus, labium majus, clitoris, vulva, overlapping lesion of vulva, penis, glans penis, prepuce, and body of penis). The exclusion criteria were as follows: 1) multiple primary cancers were excluded; 2) patients with a survival of less than one month and unknown; and 3) lack of information on tumor size, Clark level, Breslow thickness, ulceration, radiation, primary surgery site, and race (for the detailed inclusion and exclusion criteria, see Figure 1).

\section{Statistical analyses}

The eligible patients were randomly divided into a training cohort $(70 \%)$ and a validation cohort $(30 \%)$ to establish and validate a competing risk nomogram model. Continuous variables, such as age, tumor size, and Breslow thickness, were transformed into categorical variables based on recognized cutoff values. Age was classified into six groups $(\leq 29,30-39,40-49,50-59,60$ 69 , and $\geq 70$ years). Breckness thickness was divided into five groups $(<1,1-2,2-3,3-4$, and $>4 \mathrm{~mm})$. Tumor size was classified into six groups $(<1,1-2,2-3,3-4,4-5$, and $>5 \mathrm{~cm}$ ). Clark level was classified into four groups (I/II, III, IV, and V). All of the patients were regrouped according to the 6th American Joint Committee on Cancer TNM staging system. Race was divided into white, black, and other (including American Indian/AK Native, Asian/ Pacific Islander).

We defined OS as the time from diagnosis to death or censoring. The CSS was also defined as the time from diagnosis to death due to cancer-specific death or censoring. Kaplan-Meier plots and Cox regression analysis were applied to determine the prognostic factor. Variables with a $P$ value of less than 0.05 were considered independent prognostic OS factors, and the included prognostic factors were used to build the nomogram model for OS. Death from melanoma and death from other causes were two types of events in the competing risk analysis. Death from other causes was considered a competing risk [8-10]. We established competing risk analysis to acquire the cumulative incidence function (CIF) for different groups, and 3 and 5 years were recognized as cutoff times.

A nomogram model was established based on the results of the Cox proportional hazard model in the training cohort. To decrease overfit bias, the nomogram model was subjected to bootstrapping with 1000 resamples as quantified by the concordance index (C-index) for internal validation in the training cohort and external validation in the validation cohort. The value of $\mathrm{C}$-index ranges from 0.5 to 1.0 , with 0.5 suggesting no discrimination and 1.0 indicating perfect discrimination [11].

We used SPSS 19.0 software (IBM Corp., Armonk, NY, USA) to identify independent prognostic factors. Construction, validation, and calibration of the nomograms 
were developed on R version 3.1.2 software (Institute of Statistics and Mathematics, Vienna, Austria; www.r-project. org). The R "cmpprsk" and "rms" packages were used for building the model of competing analysis and nomograms, respectively. The score of the nomogram model was estimated and visualized by the "nomogramEx" package. All $P$ values were two-sided, and those less than 0.01 were considered statistically significant by a large number of patients.

\section{RESULTS}

\section{Clinical and pathological characteristics}

A total of 31,580 eligible patients were included in the study, comprising 22,101 patients in the training cohort and 9,479 patients in the validation cohort. The clinical and pathological characteristics of the cohort study are shown in Supplementary Table 1. In the whole cohort, $5.02 \%$ of patients were aged less than 29 years, $8.91 \%$ of patients were aged $30-39$ years, $16.47 \%$ of patients were aged $40-49$ years, $22.41 \%$ of patients were aged 50-59 years, $21.20 \%$ of patients were aged $60-69$ years, and $25.99 \%$ of patients were aged 70 years or older. The median age at diagnosis was 58.0 in the whole cohort. The median follow-up length was 46.0 months (range, 2-119 months). By the end of the last follow up, 7,515 (23.07\%) patients of the entire population died, including 4865 from SCM and 2650 from other causes.

Out of 22,101 patients in the training cohort, 21,721 (98.28\%) were white and 12,253 (55.44\%) were male. T3T4 tumors accounted for $19.49 \%$ of all tumors, and positive lymph nodes and distant metastases accounted for $12.05 \%$ and $1.40 \%$, respectively. Tumors were most commonly noted on the limbs $(45.72 \%)$ and trunk (34.62\%), with less than $20 \%$ arising from genitals and head and neck. The three most prevalent histologic subtypes were melanoma NOS (42.85\%), superficial spreading melanoma (36.91\%), and nodular melanomas (10.91\%). Majority of melanomas were $>4 \mathrm{~mm}$ thick, with $3.3 \%$ patients having less than $1 \mathrm{~mm}$ thick melanomas. The majority of melanomas were Clark levels II and III, whereas 6.57\% tumors were Clark level V. A total of $3701(16.75 \%)$ tumors demonstrated tumor ulceration. The majority of the tumor size of melanomas was $\leq 1 \mathrm{~cm}$, whereas $1.8 \%$ had tumor sizes of $4-5 \mathrm{~cm}$. A total of $387(1.75 \%)$ and 21,845 (98.84) patients received radiotherapy and surgery, respectively.

\section{Prognostic analyses with OS}

Our univariate analyses demonstrated that sex, age at diagnosis, TNM stage, tumor location, histologic subtype, Breslow thickness, Clark level, tumor size, radiation, and surgery of primary site were statistically associated with OS (Supplementary Table 2). For multivariate analysis, age held a significant prognostic value for OS for patients $\geq 70$ years old. Consistent with previous studies, men have poor prognosis for OS than women [4]. Tumor size, TNM stage, and Clark level were also significant independent predictors for OS. Other factors associated with poor OS included skin of genitals, tumor ulceration, and radiation. Compared with melanoma NOS histology, other histologies, except for nodular melanoma, were not associated with OS prognosis. Surgery was associated with improved OS.

\section{SCM and competing risk analysis}

According to the competing risk model, all factors excluding surgery of primary site were independent for SCM CSS $(P<0.05)$ (Supplementary Table 3$)$. At 3 and 5 years after diagnosis, the cumulative incidences of death resulting from skin melanoma in the training cohort were 0.410 and 0.506 , respectively. The cumulative incidences of death from other causes for 3 and 5 years were 0.261 and 0.359 , respectively. Estimates of death resulting from SCM and other causes by clinicopathological variables are shown in Supplementary Table 3. Patients older than 70 years at diagnosis had the highest cumulative incidences of death resulting from SCM (0.655/0.845 for 3/5 years). Male patients had the highest cumulative incidences of death resulting from skin melanoma $(0.092 / 0.124$ for $3 / 5$ years). Black patients had the highest cumulative incidences of death resulting from SCM, whereas White and "Other" (American Indian/AK Native, Asian/Pacific Islander) had lower cumulative incidences of death resulting from skin melanoma. The TNM stage also significantly influenced the prognostic factor. Patients with skin of genital and acral lentiginous melanoma had higher cumulative incidences of death resulting from SCM $(P<0.001)$. Increasing Clark level and tumor size also had higher cumulative incidences of death resulting from SCM. The CIF in different Breslow thickness groups formed a U-shaped trend, with thinness and thickness having the worst prognosis, whereas the median Breslow thickness experienced the best survival. Receiving radiation decreased cumulative incidences of death resulting from skin melanoma from $0.796 / 0.874$ for $3 / 5$ years to $0.382 / 0.479$ for $3 / 5$ years. No significant difference was found in the subgroup of surgery of the primary site. All variables, excluding surgery of the primary site, that were significantly correlated with cumulative incidences of death resulting from skin melanoma were employed to build the nomograms to predict 3- and 5-year CSS. All variables significantly correlated with SCM were used to construct the nomograms to predict 3- and 5-year probability of CSS and OS in SCM.

\section{Construction and validation of a prognostic nomogram model}

Nomograms for significant risk factors identified by the Cox model were constructed to predict the probability 
of OS and CSS in SCM in the training cohort (Figure 2). The age, race, sex, tumor site, tumor histology, TNM stage, Breslow thickness, Clark level, tumor ulceration, tumor size, radiotherapy, and surgery of primary site were included in the nomograms. The nomograms demonstrated that TNM stage, age at diagnosis, and tumor location were the largest contributors to prognosis, whereas tumor size was the lowest contributor.

We performed both internal and external validation of nomograms using the bootstrap method. Internal validation in the training cohort showed that the nomograms could accurately predict CSS with a C-index of 0.901 (95\% CI, 0.895-0.907) and OS with a C-index of 0.860 (95\% CI, $0.854-0.866)$. The calibration plots demonstrated excellent agreement between the nomogram predictions and actual observation for 3- and 5-year CSS and OS rates (Figure 3). External validation in the validation cohort indicated that the C-index was slightly low: 0.904 (95\% CI, 0.886-0.922) for CSS and 0.859 (95\% CI, 0.841-0.877) for OS (Figure 4 ). This finding suggested that the established models were reasonably accurate.

The risk score developed from the nomogram model could easily be calculated by the R "nomogramEx" package (Table 1). TNM stage, age at diagnosis, and tumor location exhibited high risk scores, whereas tumor size had a low score.

\section{DISCUSSION}

SCM is the most dangerous type of cutaneous malignant tumor with considerable risk of developing distant and lymph metastases $[12,13]$. The poor prognosis and clinical outcome are due to the poorly effective therapeutic methods currently available. Therefore, further understanding of the risk factors underlying SCM development is necessary to identify new early diagnostic factors and therapeutic targets. Prior studies evaluated the survival difference among different anatomical sites and histologic subtypes [14, 15]. Considering other clinicopathologic characteristics like age, race, sex, tumor site, tumor histology, TNM stage, Breslow thickness, Clark level, tumor ulceration, tumor size, and treatment which were associated with SCM prognosis, is necessary for predicting prognostic analyses. A recent non-metastatic SCM study based on the SEER database between 2004 and 2007 constructed nomograms, but Breslow thickness, Clark level, tumor ulceration, and tumor size were unavailable in the study, and the followup period was relatively short [16]. Using the SEER database, we screened the patients with SCM between 2003 and 2013, and 14 clinicopathologic characteristics (age, race, sex, tumor site, tumor histology, TNM stage, Breslow thickness, Clark level, tumor ulceration, tumor size, radiotherapy, and surgery of primary site) were considered in the study. The patients with SCM had significantly higher 3- and 5-year OS and CSS death probabilities ( 0.410 and 0.506 , respectively) than those without SCM, and nomograms were established to predict the 3- and 5-year CSS and OS on the basis of competing risk analysis.

Several clinicopathological characteristics were proven to be independent prognosis factors for both

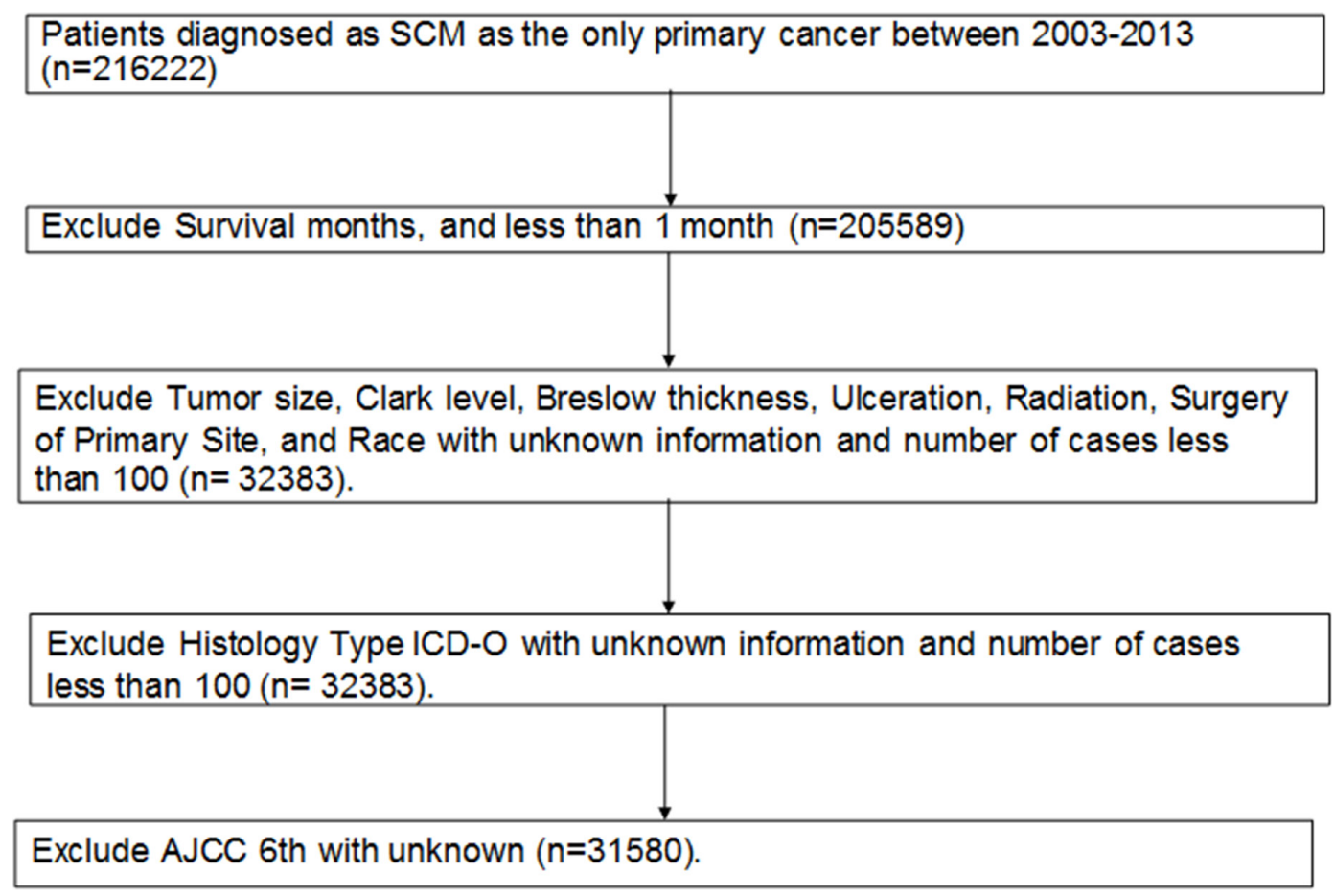

Figure 1: Flow chart for the SEER data selection. 


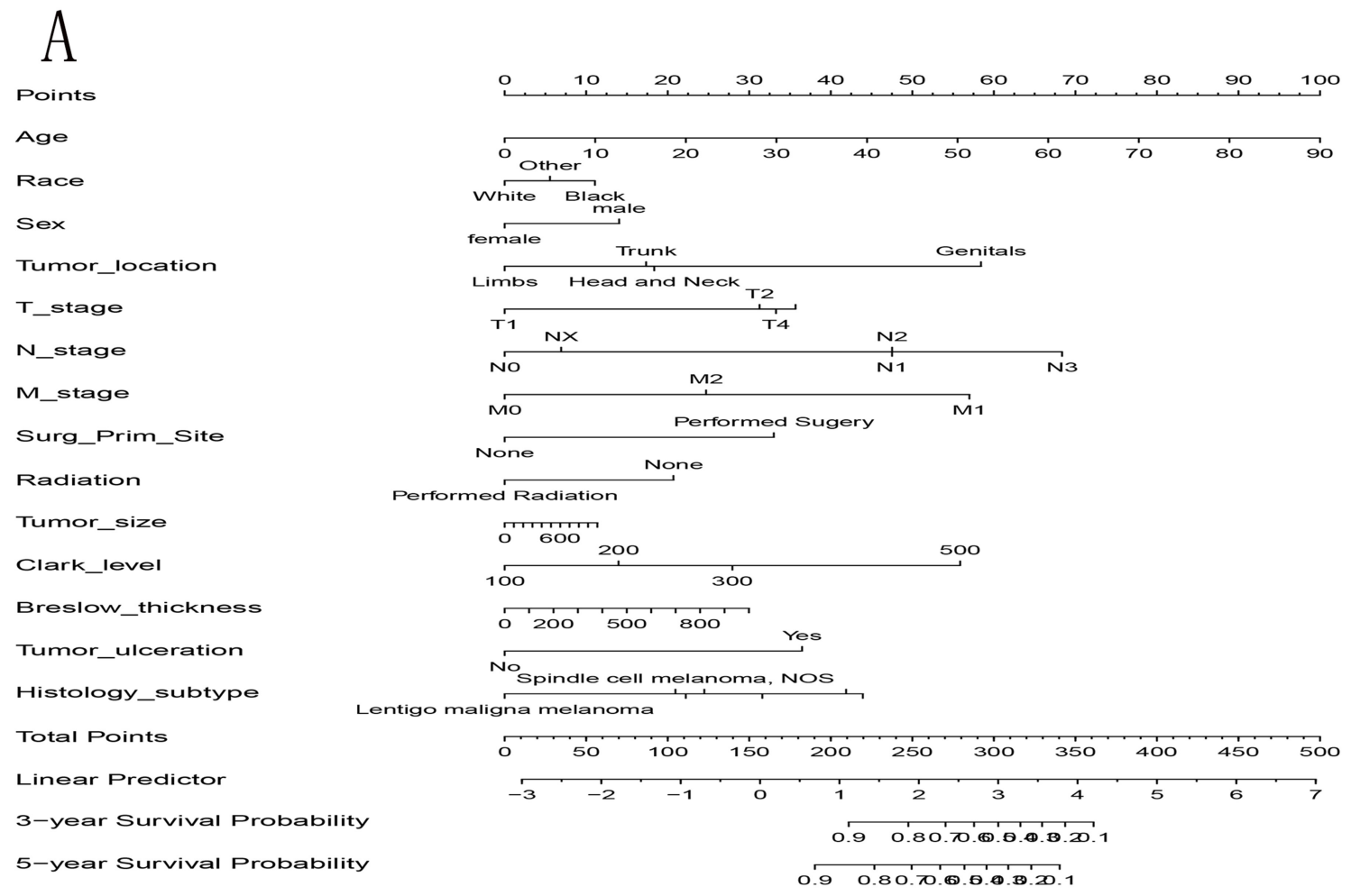

\section{B}

Points

Age

Race

Sex

Tumor_location

T_stage

N_stage

M_stage

Surg_Prim_Site

Radiation

Tumor_size

Clark_level

Breslow_thickness

Tumor_ulceration

Histology_subtype

Total Points

Linear Predictor

3-year Survival Probability

5-year Survival Probability

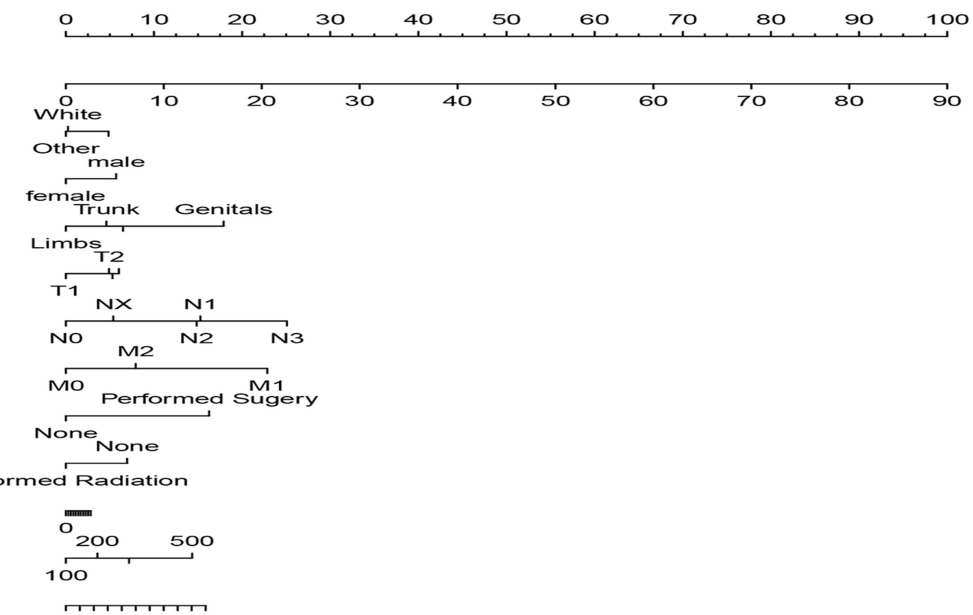

Spindle cell melanoma, NOS

Desmoplastic melanoma, malignant

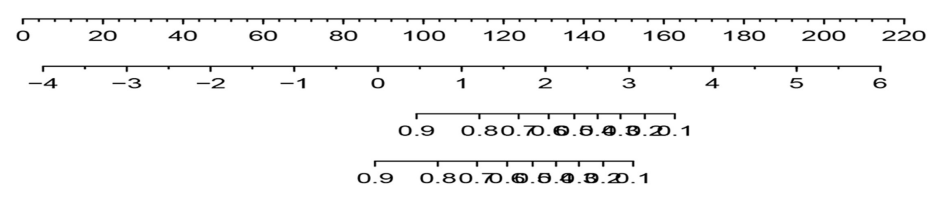

Figure 2: Nomograms for estimating 3- and 5- year survival. (A) overall survival (OS) and (B) cancer-specific survival (CSS) in patients with SCM. 
OS and CSS in the present study, including age, race, sex, tumor site, tumor histology, TNM stage, Breslow thickness, Clark level, tumor ulceration, tumor size, radiotherapy, and surgery of the primary site. Next, we used the log-rank test and Cox proportional hazards
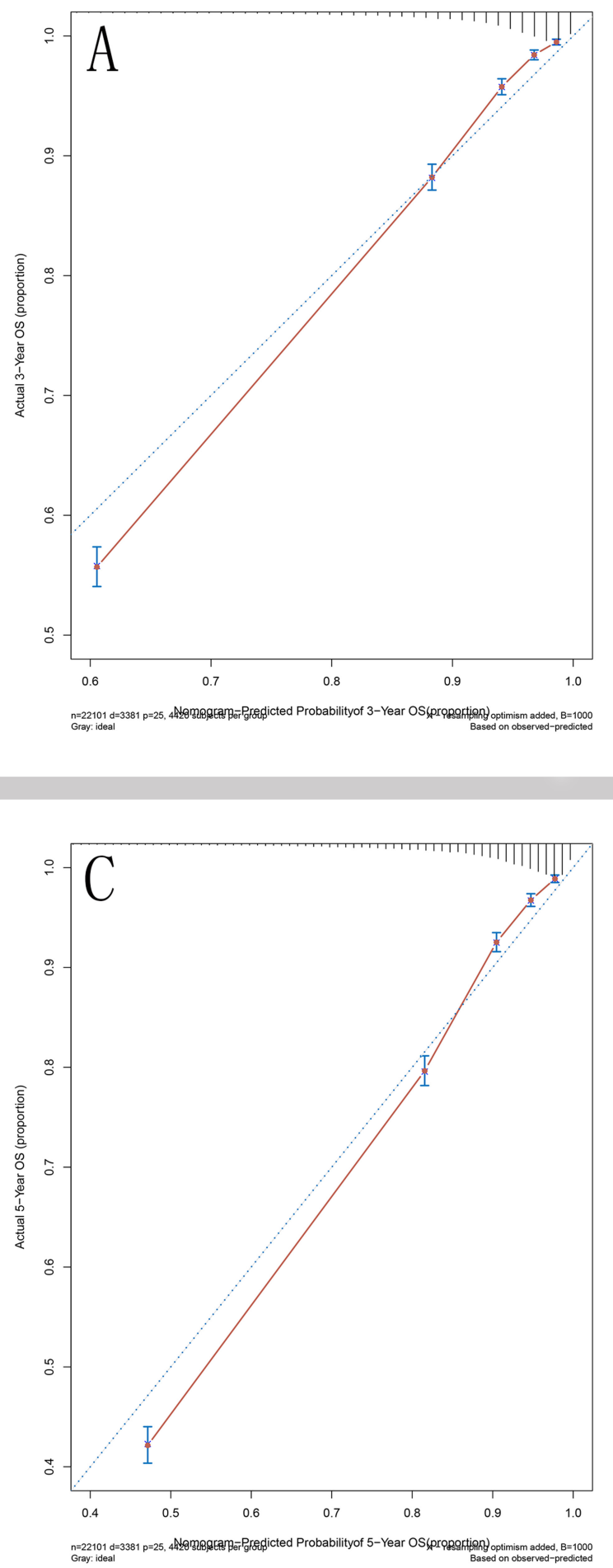

regression to screen the independent prognosis factors of OS. However, these results could not be used to identify prognostic variables of SCM because death from other causes was considered as a competing risk event. Therefore, a competing risk was employed to avoid
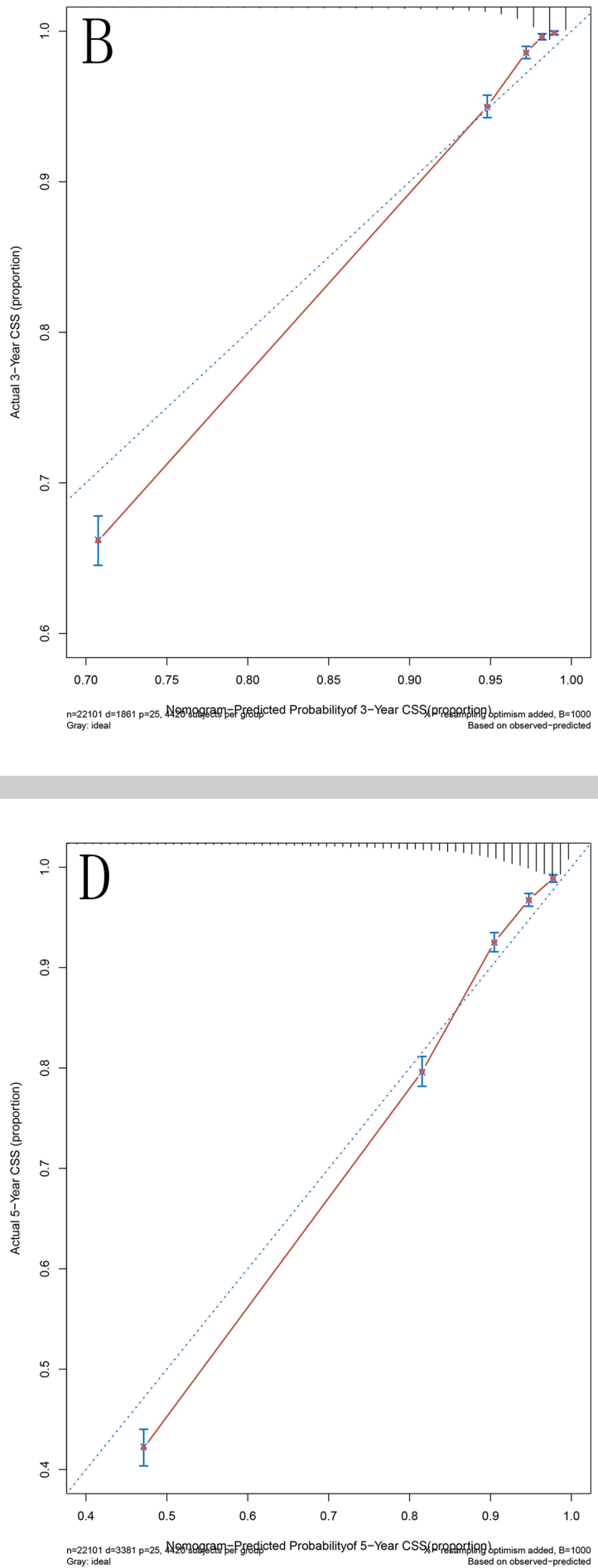

Figure 3: Internal calibration of the nomograms. (A) 3- and (C) 5- year overall survival (OS) calibration curves; (B) 3- and (D) 5- year cancer-specific survival (CSS) calibration curves. 
biased results. As shown in this study, the 3- and 5-year cumulative incidences of death resulting from SCM were 0.410 and 0.506 , respectively. These findings indicated that more patients died due to SCM during the subsequent two years. These results were consistent with previous studies $[14,15]$. Among these risk factors, age at diagnosis was an important prognostic factor. TNM stage, Breslow thickness, Clark level, and tumor size were also associated with increased CIF resulting from SCM death. Consistent with previous studies, the gender-specific disparity in
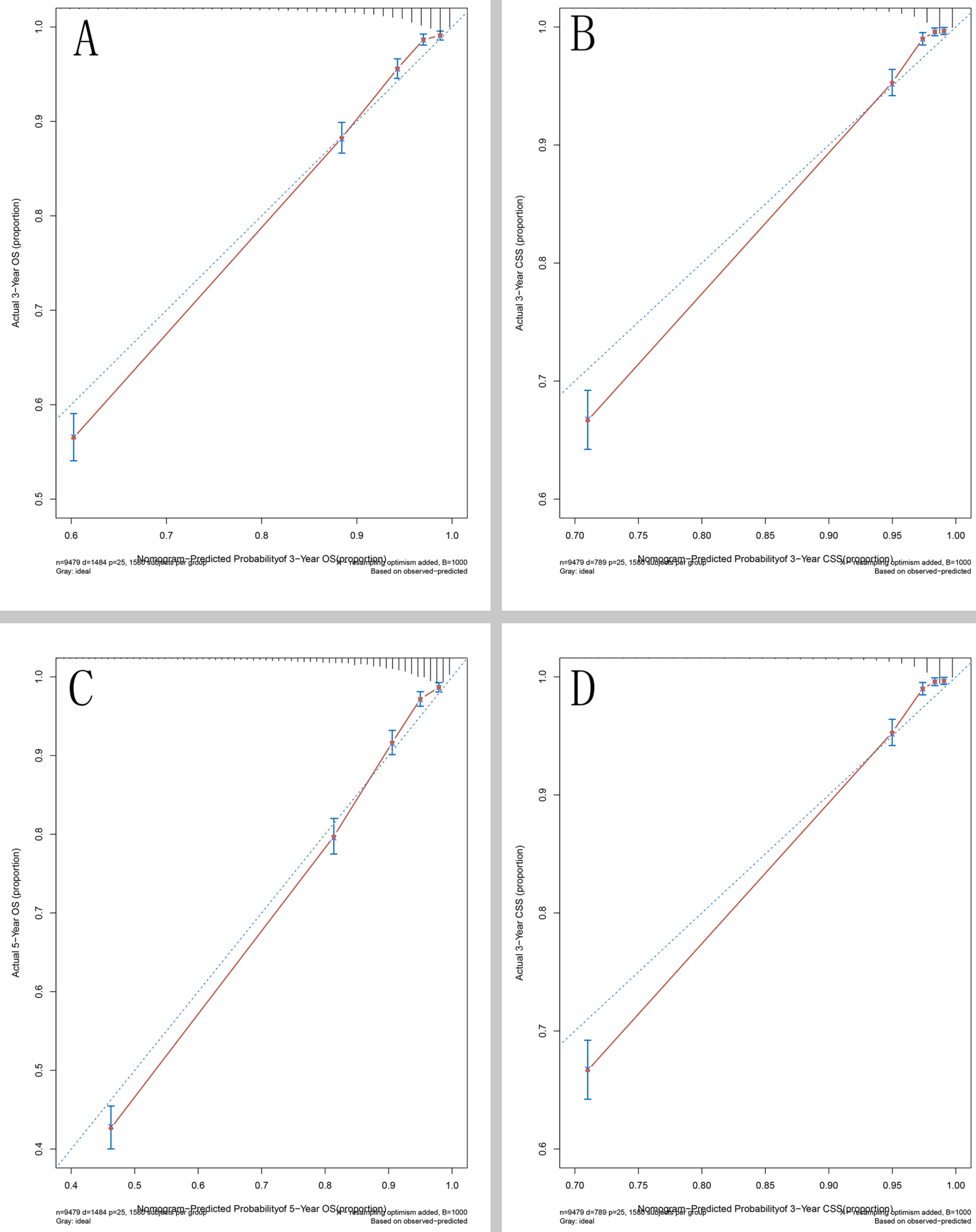

Figure 4: External calibration of the nomograms. (A) 3- and (C) 5- year overall survival (OS) calibration curves; (B. 3- and D) 5- year cancer-specific survival (CSS) calibration curves. 
Table 1: Prognostic score for OS and CSS in the Nomograms plot

\begin{tabular}{|c|c|c|}
\hline Variables & OS & CSS \\
\hline \multicolumn{3}{|l|}{ Sex } \\
\hline Female & 0.000 & 0.000 \\
\hline Male & 6.878 & 22.548 \\
\hline \multicolumn{3}{|l|}{ Age at diagnosis, years } \\
\hline Equation & $1.111^{*} \mathrm{Age}$ & $1.111^{*} \mathrm{Age}$ \\
\hline \multicolumn{3}{|l|}{ Race } \\
\hline White & 0.000 & 0.000 \\
\hline Black & 1.370 & 7.617 \\
\hline Other (American Indian/AK Native, Asian/Pacific Islander) & 2.741 & 15.547 \\
\hline \multicolumn{3}{|l|}{ T stage } \\
\hline $\mathrm{T} 1$ & 0.000 & 0.000 \\
\hline $\mathrm{T} 2$ & 3.514 & 22.934 \\
\hline $\mathrm{T} 3$ & 7.028 & 45.869 \\
\hline $\mathrm{T} 4$ & 10.542 & 68.803 \\
\hline \multicolumn{3}{|l|}{ N stage } \\
\hline N0 & 0.000 & 0.000 \\
\hline N1 & 5.081 & 18.299 \\
\hline N2 & 10.163 & 36.599 \\
\hline N3 & 15.244 & 54.899 \\
\hline NX & 20.326 & 73.199 \\
\hline \multicolumn{3}{|l|}{ M stage } \\
\hline M0 & 0.000 & 0.000 \\
\hline M1 & 4.178 & 18.033 \\
\hline MX & 8.356 & 36.067 \\
\hline \multicolumn{3}{|l|}{ Tumor location } \\
\hline Skin of head and neck & 6.468 & 16.232 \\
\hline Skin of trunk & 4.312 & 10.821 \\
\hline Skin of limbs & 2.156 & 5.411 \\
\hline Skin of genitals & 0.000 & 0.000 \\
\hline \multicolumn{3}{|l|}{ Histologic subtype } \\
\hline Malignant melanoma, NOS & 0.000 & 0.000 \\
\hline Nodular melanoma & 5.345 & 18.948 \\
\hline Lentigo maligna melanoma & 4.276 & 15.981 \\
\hline Superficial spreading melanoma & 3.207 & 11.370 \\
\hline Acral lentiginous melanoma, malignant & 2.318 & 7.581 \\
\hline Desmoplastic melanoma, malignant & 1.069 & 3.792 \\
\hline Spindle cell melanoma, NOS & 0.000 & 0.000 \\
\hline \multicolumn{3}{|l|}{ Breslow thickness } \\
\hline Equation & $0.014^{*}$ Breslow thickness & $0.014^{*}$ Breslow thickness \\
\hline \multicolumn{3}{|l|}{ Clark level } \\
\hline II & 0.000 & 0.000 \\
\hline III & 4.8130 & 22.694 \\
\hline IV & 9.626 & 45.388 \\
\hline $\mathrm{V}$ & 19.252 & 90.777 \\
\hline
\end{tabular}




\begin{tabular}{|c|c|c|}
\hline \multicolumn{3}{|c|}{ Tumor ulcertion } \\
\hline Yes & 0.000 & 0.000 \\
\hline No & 15.181 & 59.619 \\
\hline \multicolumn{3}{|c|}{ Tumor size } \\
\hline Equation & $0.001^{*}$ tumor size & $0.008^{*}$ tumor size \\
\hline \multicolumn{3}{|l|}{ Radiotion } \\
\hline No & 0.000 & 0.000 \\
\hline Yes & 15.871 & 52.618 \\
\hline \multicolumn{3}{|c|}{ Surgery of primary site } \\
\hline No & 17.720 & 45.692 \\
\hline Yes & 0.000 & 0.000 \\
\hline
\end{tabular}

SCM revealed that men had a higher CIF value than women $[17,18]$. The causes of gender difference in SCM remain unclear but are likely due to estrogen [19, 20], pregnancy [21, 22], oral contraceptives [23], and hormone replacement therapy [24].

Both the 3- and 5-year CSSs of patients with SCM who received radiotherapy $(0.382$ and 0.479 , respectively) were lower than those of patients with SCM who did not receive radiotherapy. Meanwhile, the CIF of death resulting from other causes increased with radiotherapy. By contrast, the 3- and 5-year death rates by other causes of patients with SCM who received radiotherapy were higher than those of patients with SCM without radiotherapy. Radiotherapy was associated with poor OS probability. This phenomenon indicated that radiotherapy might be associated with increased death from other causes. Interestingly, we found that the 3-and 5-year cause-specific death rates of SCM who did not receive surgery of the primary site ( 0.409 and 0.507 , respectively) were higher than those of SCM who died of other causes. The detailed mechanism was unclear.

The prognostic nomograms were based on a modelbased prediction tool and incorporated clinical and pathologic risk factors known to influence the outcome. To guarantee the prediction accuracy of nomograms, Cox's proportional hazards regression model was used to select the variables. The nomograms indicated that age at diagnosis, tumor size, and TNM stage showed significant predictive capability for the prognosis of patients with SKCM. In the nomogram plot, the hazard ratios of OS and CSD in different age groups produced a linear association, with older patients suffering the worst survival rate. Other clinical and pathological factors, including race, sex, tumor site, tumor histology, Breslow thickness, Clark level, tumor ulceration, radiotherapy, and surgery of the primary site, were also associated with increased SCM mortality.

The present study exhibited several strengths. First, the greatest strength was the large cohort size and high quality of the SEER database. The conclusions from a population-based study were more reliable than those from a single study because the study was based on a large sample size and exhibited sufficient power. In addition, the large sample size was sufficient to allow a predictive model to be built accurately. Second, the Cox's proportional hazards regression model and competing risk analysis were used to screen the variables. All C-indexes of the nomograms were greater than 0.7. Excellent agreement between the calibration curve and 45-degree perfect match straight lines was observed, suggesting that the established model had good accuracy for predicting OS and CSD.

Despite these strengths, the study had some limitations. First, the study was based on a retrospective design that produced some bias, such as recall bias and selective bias. These results must be further validated in a prospective cohort before being applied to clinical use. Second, information regarding adjuvant therapy, such as chemotherapy, endocrine therapy, and targeted therapy, which are important prognostic risk factors for SCM $[25,26]$, was unavailable in the SEER database. Without the information, the nomograms might yield some bias. Finally, external validation of the nomograms was not performed in our study.

In summary, we developed nomograms to estimate the probability of OS and CSS of SCM on the basis of a population-based cohort with long-term follow up. The constructed nomograms could help clinicians predict individual prognosis with SCM, thereby providing more individualized treatment strategies.

\section{CONFLICTS OF INTEREST}

None.

\section{GRANT SUPPORT}

The study was supported by the National Science Foundation of China (No. 81700972) and Joint fund project of Guizhou science and Technology Department (No. [2014] 7026). 


\section{REFERENCES}

1. Purdue MP, Freeman LE, Anderson WF, Tucker MA. Recent trends in incidence of cutaneous melanoma among US Caucasian young adults. The Journal of investigative dermatology. 2008; 128:2905-2908.

2. Jemal A, Saraiya M, Patel P, Cherala SS, Barnholtz-Sloan J, Kim J, Wiggins CL, Wingo PA. Recent trends in cutaneous melanoma incidence and death rates in the United States, 1992-2006. Journal of the American Academy of Dermatology. 2011; 65:S17-25 e11-13.

3. Siegel RL, Miller KD, Jemal A. Cancer Statistics, 2017. CA: a cancer journal for clinicians. 2017; 67:7-30.

4. Peterson M, Albertini MR, Remington P. Incidence, Survival, and Mortality of Malignant Cutaneous Melanoma in Wisconsin, 1995-2011. WMJ. 2015; 114:196-201.

5. Insinga RP, Reither EN, Remington PL, Stephenson-Vine L. Trends in malignant melanoma incidence and mortality in Wisconsin, 1979-1997. WMJ. 2001; 100:27-31.

6. Zeng C, Wen W, Morgans AK, Pao W, Shu XO, Zheng W. Disparities by Race, Age, and Sex in the Improvement of Survival for Major Cancers: Results From the National Cancer Institute Surveillance, Epidemiology, and End Results (SEER) Program in the United States, 1990 to 2010. JAMA oncology. 2015; 1:88-96.

7. Hankey BF, Ries LA, Edwards BK. The surveillance, epidemiology, and end results program. Cancer Epidemiology and Prevention Biomarkers. 1999; 8:1117-1121.

8. He P, Eriksson F, Scheike TH, Zhang MJ. A Proportional Hazards Regression Model for the Sub-distribution with Covariates Adjusted Censoring Weight for Competing Risks Data. Scandinavian journal of statistics, theory and applications. 2016; 43:103-122.

9. Gray RJ. A class of K-sample tests for comparing the cumulative incidence of a competing risk. The Annals of statistics. 1988; 16:1141-1154.

10. Fine JP, Gray RJ. A proportional hazards model for the subdistribution of a competing risk. Journal of the American statistical association. 1999; 94:496-509.

11. Harrell FE. Regression modeling strategies: with applications to linear models, logistic regression, and survival analysis. New York; Springer-Verlag: 2001.

12. Watson M, Geller AC, Tucker MA, Guy GP Jr, Weinstock MA. Melanoma burden and recent trends among nonHispanic whites aged 15-49years, United States. Preventive medicine. 2016; 91:294-298.

13. White WL, Hitchcock MG. Dying dogma: the pathological diagnosis of epidermotropic metastatic malignant melanoma. Seminars in diagnostic pathology. 1998; 15:176-188.
14. Tseng WH, Martinez SR. Tumor location predicts survival in cutaneous head and neck melanoma. The Journal of surgical research. 2011; 167:192-198.

15. Jethanamest D, Vila PM, Sikora AG, Morris LG. Predictors of survival in mucosal melanoma of the head and neck. Annals of surgical oncology. 2011; 18:2748-2756.

16. Shen W, Sakamoto N, Yang L. Melanoma-specific mortality and competing mortality in patients with non-metastatic malignant melanoma: a population-based analysis. BMC cancer. 2016; 16:413.

17. Roh MR, Eliades P, Gupta S, Grant-Kels JM, Tsao H. Cutaneous melanoma in women. International journal of women's dermatology. 2015; 1:21-25.

18. Tremblay GB, Tremblay A, Copeland NG, Gilbert DJ, Jenkins NA, Labrie F, Giguere V. Cloning, chromosomal localization, and functional analysis of the murine estrogen receptor beta. Molecular endocrinology. 1997; 11:353-365.

19. Hall G, Phillips TJ. Estrogen and skin: the effects of estrogen, menopause, and hormone replacement therapy on the skin. Journal of the American Academy of Dermatology. 2005; 53:555-568; quiz 569-572.

20. Ohata C, Tadokoro T, Itami S. Expression of estrogen receptor beta in normal skin, melanocytic nevi and malignant melanomas. The Journal of dermatology. 2008; 35:215-221.

21. Lens M. Melanoma during pregnancy: epidemiology, diagnosis, staging, clinical picture. Recent results in cancer research. 2008; 178:165-174.

22. Daryanani D, Plukker JT, De Hullu JA, Kuiper H, Nap RE, Hoekstra HJ. Pregnancy and early-stage melanoma. Cancer. 2003; 97:2248-2253.

23. Gefeller O, Hassan K, Wille L. Cutaneous malignant melanoma in women and the role of oral contraceptives. The British journal of dermatology. 1998; 138:122-124.

24. Persson I, Yuen J, Bergkvist L, Schairer C. Cancer incidence and mortality in women receiving estrogen and estrogenprogestin replacement therapy--long-term follow-up of a Swedish cohort. International journal of cancer. 1996; 67:327-332.

25. Aguado-Romeo MJ, Benot-Lopez S, Romero-Tabares A. Electrochemotherapy for the Treatment of Unresectable Locoregionally Advanced Cutaneous Melanoma: A Systematic Review. Actas dermo-sifiliograficas. 2017; 108:91-97.

26. Stoica A, Hoffman M, Marta L, Voiculetz N. Estradiol and progesterone receptors in human cutaneous melanoma. Neoplasma. 1991; 38:137-145. 